\title{
Motivational Media and Academic Success
}

\author{
Nicolle Grant ${ }^{\mathrm{a}}$
}

Motivating students to do well in courses that fail to interest them can be challenging. The current study was designed to assess the potential impact of priming motivational media on academic performance. Students in six sections of introductory psychology courses $(n=128)$ completed a quiz after watching a 4 minute inspirational video (experimental group, $n=63$ ), or without watching the video (control group, $n=65$ ). The video selected showed scenes of individuals pushing their limits in all aspects of life and a commentary of a motivational commencement speech by Arnold Schwarzenegger given to college graduates. After completing the quiz participants were given a survey with questions pertaining to their motivation, positive and negative emotions, demographics, and their reactions to the video. Students who were primed by the video reported greater academic motivation and planned to study more for the next quiz than students who did not watch the video. Females reported lower GPA and lower positive emotions in the experimental condition. While there was not an overall effect of the video on quiz scores, male students who watched the video performed $10 \%$ better on the quiz than males in the control condition. Implications of these findings suggest that motivational information may be beneficial in inspiring academic performance in certain circumstances.

Keywords: Motivation; academic performance; priming

\section{Introduction}

It can seem surprising that students may need to be motivated when they have chosen to enroll in an academic institution such as a university or college, but when taking courses they may not think are relevant, motivating them may be imperative for their success. Motivation is induced either intrinsically or extrinsically depending on the goals that have been set. Intrinsic motivation arises when the individual has no end goal or reward but rather completes a task for their personal enjoyment and interest (Berlyne, 1960). When a student is motivated to complete a task in an academic course purely because they enjoy the subject or challenge, they are engaging in intrinsic motivation. Extrinsic motivation occurs when a reward is attained once a task is complete. The individual is motivated externally to complete a task because of the reward and not because of personal enjoyment and fulfillment. For many students, extrinsic motivation is what drives them to study vigorously for years. The excitement and satisfaction of attaining a degree can be enough to motivate college students, but this may not be sufficient for first or second year students who still have some time until they attain a degree. To motivate these students, they may need to be reminded of their goals and the process of achieving those goals. The process may include smaller goals which may appear trivial, such as quizzes and homework assignments. An individual's behavior toward a set goal may predict their success at achieving that goal in the future (Gramzow, 2014). A reminder of the consequences, either good or bad, for these small tasks may result in a beneficial higher arousal level.

Research on students in academic institutions has determined how mood and emotion can affect motivation and performance. Some research in particular relates to how music can alter students' moods and as a result change how they perform in class. McConnell (2011) studied the interactions and effects of music on emotion and attention, with the intent that certain music pieces would induce either positive or negative emotions in students and ultimately affect their performances. The music produced either high or low arousal levels based on the tempo and mode of the music piece. Although the results were somewhat discrete in their interactions, fast-tempo music did seem to produce more positive emotions in the students than the slow-tempo music (2011). The results from this study provide us with an understanding of how priming can affect academic performance and to investigate the different priming agents that can help students become more motivated.

Another similar study, conducted by Hepler (2012), showed that priming individuals increased their motivation to exercise. The study involved 111 undergraduate students and was asked to complete a task on their individual computers. The computer task was a virtual game where a ball is thrown between players. Individuals either participated in the computer game or they observed the game being played virtualy. The individuals were primed after the computer task with words that were either action words or inaction words and then were instructed to complete a series of exercises that they were able to complete while seated. The results showed that individuals who watched the game being played on the computer exercised longer than those who actually participated in it. Further, they found that participants who observed the actions and then received action words exercised more. With these results, we can see that priming agents may benefit students in becoming more motivated in academic situations.

Priming can change and affect behaviors in academic performance on a long term basis. Lowery (2007) studied this possibility by showing neutral and intelligence words to students a few days before they completed a practice exam. Seventy undergraduate students participated in the study and were recruited from an introductory statistics course. Lowery found that when students were primed with intelligence words before they completed a practice exam a few days later, they did significantly better than if they were shown neutral words. This previous research suggests that students' behavior can be changed and affected by priming agents even on a long-term basis. We can see this change in athletes as they listen to or watch motivational videos, which serve as the priming agent, before they compete in their sport (Sturmey, 2007). If athletes 
can be positively affected by motivational media, students in academic institutions may have the same positive results.

In this current study, we used motivational media as a priming instrument and predicted that students would be more motivated after watching motivational images. We hypothesized that students in the experimental group would perform better on a quiz than students in the control group because the video would inspire them to perform at their best levels. Students would also report greater academic motivation in the experimental group than students in the control group, resulting in better academic performance. We also hypothesized that students who reported that they failed to study sufficiently for the quiz in the experimental group will perform worse on the quiz and report more negative emotions compared to those who failed to study for the quiz in the control group, because they would be reminded of the fact that they never studied and therefore be negatively affected by the video.

\section{Methods}

\section{Participants}

Students from six sections of introductory psychology classes $(n=128)$ participated in this study. There were 65 female and 60 male participants (3 participants did not specify their gender). The majority $(65 \%)$ of the participants were student athletes. The average of age of the students was 19.6 and the average GPA was 3.14. Most of the participants were white (75 out of 128), followed by African American (39) and Hispanic/Latino (4). Two participants were of another race not specified and 8 students did not state their race.

\section{Procedure}

Students were recruited from six sections of entry level psychology courses at Limestone College. These courses included two sections of Human Growth and Development, two sections of Psychology of Adjustment and two sections of Introduction to Psychology. The students were given a 10 point quiz in accordance with the material they covered in the specific classes the students were enrolled in. Attached to the quiz was a form of consent and a survey for each student to fill out. Half of the students watched a 4 minute motivational video before they took the quiz while the other half were not shown the video but completed the same quizzes. Each class had both a control condition and an experimental condition (for example, one section of Introduction to Psychology watched the video and one section did not).

All participants completed a survey after the quiz with questions pertaining to the video, if they watched it, and to their motivation and goals to study more for the next quiz. The students in the experimental group answered 5 questions related to the video and how it may have impacted them. Example questions that were included in the experimental group included "Does the video inspire you to perform better academically in the future?" and "Do you think the video positively affected your performance on the quiz?" Example survey questions for both the control and experimental groups included "Do you plan to study more for the next quiz in this class?" and "Do you wish you had studied more for today's quiz?"

The video selected showed scenes of accomplishment and competition with an inspirational clip from a commencement speech by Arnold Schwarzenegger. The video was 4 minutes 30 seconds long and was a compilation of both athletic and general life instances. For example, a man was drawing a timeline of his goal and what it might take to achieve that. An example from Arnold's speech is "You never want to fail because you didn't work hard enough."

\section{Results}

To examine whether watching a motivational video impacted student quiz scores, a t-test was conducted. No significant differences were found between student quiz scores in the control condition versus student quiz scores in the experimental condition. However, an examination within gender determined that males scored significantly higher $(t(64)=2.33, p=.023)$ on the quiz in the video condition $(M$ $=74.1, S D=1.74)$ than in the control condition $(M=63.6, S D$ $=1.87$ ). See Figure 1 below.

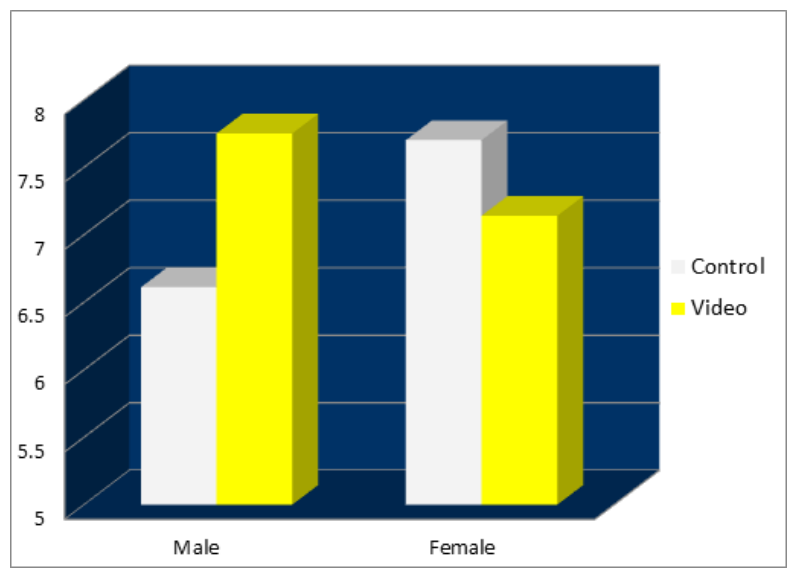

Figure 1: Quiz Scores for males and females

To examine whether watching a motivational video impacted student motivation and plans to study in the future, a t-test was conducted. Students reported significantly higher $(t$ $(127)=2.30, p=.023)$ motivation in the video condition $(M=$ $3.66, S D=1.00)$ than in the control condition $(M=3.29, S D=$ 1.02). Students reported significantly higher $(t(127)=2.07, p$ $=.04)$ plans to study in the video condition $(M=4.60, S D=$ $1.00)$ than in the control condition $(M=3.97, S D=0.68)$. See Figure 2 below.

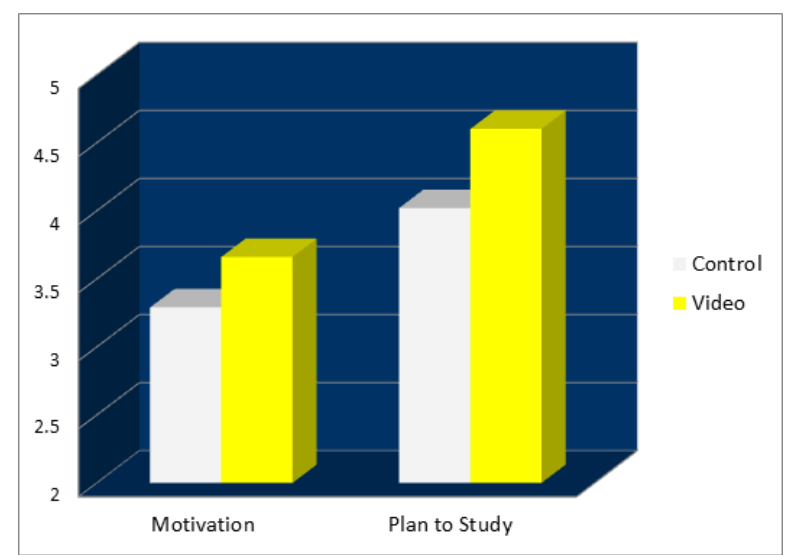

Figure 2: Motivation and plan to study for next quiz 
Further analysis on gender differences revealed an interesting interaction: Females had less positive emotions (focused, confident, motivated, satisfied) in the video condition $(M=11.47, S D=3.73)$ than in the control condition $(M=13.33, S D=3.17, t(63)=2.09, p=.04)$, whereas men had higher positive emotions in the video condition $(M=$ $13.66, S D=2.90)$ than in the control condition $(M=11.76$, $S D=2.77, t(59)=2.70, p=.009)$. Interestingly, females also reported a lower GPA in the video condition $(M=3.08, S D=$ $0.51)$ than in the control condition $(M=3.39, S D=0.50, t(59)$ $=2.75, p=.008$ ). See Figure 3 .

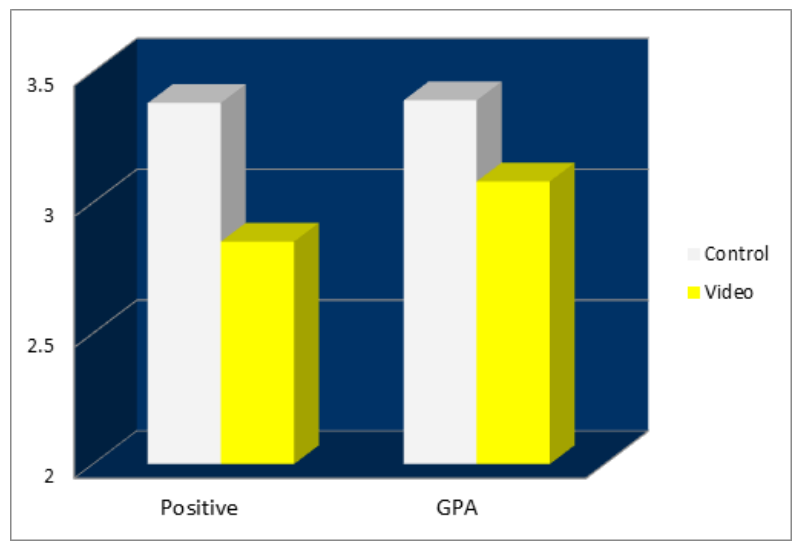

Figure 3: Female positive emotions and GPA

To examine whether students who reported that they did not study for the quiz experienced more negative emotions (disappointed, embarrassed, bored, guilty, indifferent, and anxious) after watching the video, a t-test revealed no overall main effect. However, students who did not study reported greater boredom $(M=2.25, \mathrm{SD}=1.06$, vs $M=1.56, S D=$ $0.81, t(37)=2.12, p=.04)$ and disappointment $(M=2.57, S D$ $=1.33$, vs $M=1.73, S D=0.80, t(37)=2.18, p=.036)$ in the control condition than in the experimental condition. See Figure 4.

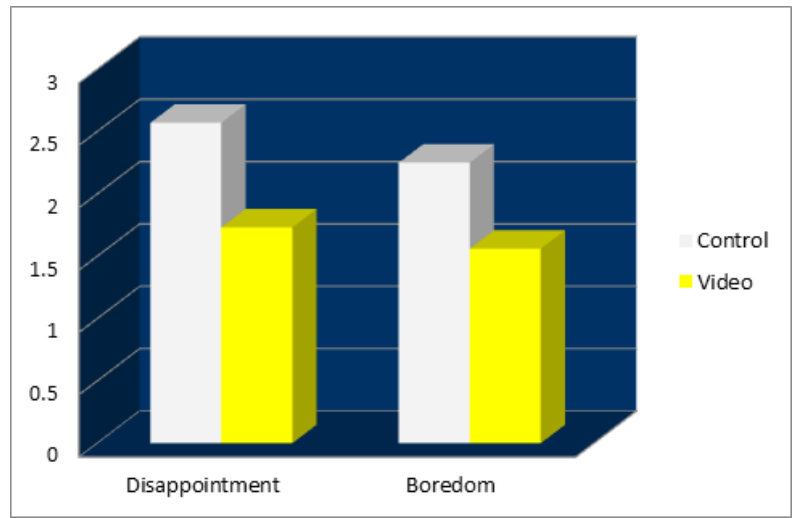

Figure 4: Students who did not study

\section{Discussion}

Participants reported greater motivation and planned to study more in the future if they were in the experimental group rather than the control group. As predicted, the video seemed to positively affect most students. We hypothesized that students who did not study for the quiz in the control group would feel less disappointment, less anxious and less guilty about their performance on the quiz than those who did not study in the experimental group. However, the results showed that students who did not study sufficiently for the quiz felt less disappointment and less boredom. This shows that the video made the participants feel better about their performance regardless of whether they studied or not.

Although we did not find a main effect of the video on quiz scores in the experimental group, we did find that males in the experimental group performed over $10 \%$ better than males in the control condition. Male students in the experimental group scored significantly better on the quiz $(74.1 \%)$ than male students in the control group (63.6\%). Females did not show a significant change in test scores between the two groups. This shows that the video positively impacted the male students but interestingly had no effect on female students.

However, female participants reported a lower GPA, and lower average positive emotions if they were in the experimental group. This result was contradictory to the males who were positively affected by the video. A possible reason for this distinction would be that the females who watched the video may have been more honest about their GPA scores than the males who watched the video (Gramzow, 2014).

\section{Considerations}

The differences between the male and female performance was surprising as we did not predict that the female students would be negatively affected by the video. This could be a result of many variables that we were unable to account for before the study. One such variable is the video itself. It may have reminded the female students about the fear of failure and that success is mostly viewed as a male attribution in society (O'Connell, 1984). Males may have done better after watching the video because instead of focusing on not failing, they may have been more focused on doing well on the quiz.

Another variable could have been the speaker in the video, Arnold Schwarzenegger. As an actor, Arnold is most commonly known for roles that include action and violence, something that appeals to male students more than females. Fischoff (1998) studied the differences in female and male film choices and found that films that appealed to males focused more on action, sex and competition while females preferred films that were more story based and told from a woman's point of view. This may have been an unconscious association that the students made and ultimately affected the females and males in different ways.

Another difficulty in determining the results, was the fact that there were three different psychology courses which meant three different types of quizzes and instructors. Teaching styles and gender differences can contribute to students' performance and may affect their quiz scores. The quizzes were administered in different ways, for one course it 
was an open book while the other two courses it was closed book. Another consideration would be the type of school that this study was conducted at where the majority of students are student-athletes $(65 \%)$. This type of video may appeal more to student-athletes who have used similar methods when they participate in sports.

Implications

This study was intended to find possible ways that students can be motivated academically in a college which consists predominantly of student-athletes. We found positive results that can be used as a basis for further research at Limestone College as well as other universities and colleges. From the results we obtained from this study, it is possible that similar methods could be used in middle schools and high schools.

The results we got from this study indicate that a more longitudinal study be set in place to account for the different variables mentioned previously. A follow up study of how the students performed on later quizzes and exams after watching a motivational video could provide more information about how the students were impacted by the video. To account for the gender differences in the experimental group, a video may need to be created that is specific to an academic setting and gender type. For instance, to see if females are affected by motivational media in a more positive manor, we might want to show a more feminine video and record their reactions compared to when they watch a more masculine video.

\section{Acknowledgements}

I would like to thank the Limestone College Psychology Department for their assistance in this study. Special thanks to Dr. Justin Bailey and Austin Gregg.

\section{References}

Berlyne, D. E. (1960). Conflict, arousal and curiosity. McGraw-Hill series in psychology. New York, NY, US: McGraw-Hill Book Company, xii, 350 pp.

Deckers, L. (2001). Motivation: Biological, psychological, and environmental. Boston, MA: Allyn and Bacon.

Fischer, S., Antonio, J., \& Lewis, D. (1998). Favorite Films and Film Genres as a Function of Race, Age, and Gender. Journal of Media Psychology, 3(1).

Gramzow, R., Johnson, C., \& Willard, G. (2014). Boasts are a boost: Achievement prime self-reactivity predicts subsequent academic performance. Journal of Personality and Social Psychology, 106(3), 458-468. http://dx.doi.org/10.1037/a0035560

Hepler, J. (2012). Motivating exercise: The interactive effect of general action goals and past behavior on physical activity. Motivation \& Emotion, 36(3), 365-370.

Lowery, B. (2007). Long-term Effects of Subliminal Priming on Academic Performance. Basic \& Applied Social Psychology, 29(2), 151-157

McConnell, M. I. (2011). Upbeat and happy: Arousal as an important factor in studying attention. Cognition \& Emotion, 25(7), 1184-1195.

O'Connell, A. (1982). Fear of success and casual attributions of success and failure in high school and college students. Journal of Psychology, 111(1), 141.

Sturmey, P. (2007). Behavioral case fromulation and intervention: A functional analytic approach. Hoboken, NJ: John Wiley \& Sons. 